\title{
UNIFORM MANUFACTURER-INDEPENDENT Mobile Robot Programming
}

\author{
Lucas Muster $^{1}$, Wilfried Woeber ${ }^{2} \&$ Mohamed Aburaia $^{3}$ \\ ${ }^{1}$ Lucas Muster is with the Department of Industrial Engineering, University of Applied Sciences Technikum Wien, \\ 1200 Vienna, Austria lucas.muster@technikum-wien.at
}

\begin{abstract}
${ }^{2}$ Wilfried Wöber is with the Department of Industrial Engineering, University of Applied Sciences Technikum Wien, 1200 Vienna, Institute for Integrative Nature Conservation Research, University of Natural Resources and Life Sciences, Vienna Austria wilfried.woeber@technikum-wien.at
\end{abstract}

${ }^{3}$ Mohamed Aburaia is with the Department of Industrial Engineering, University of Applied Sciences Technikum Wien, 1200 Vienna, Austria mohamed.aburaia@technikum-wien.at
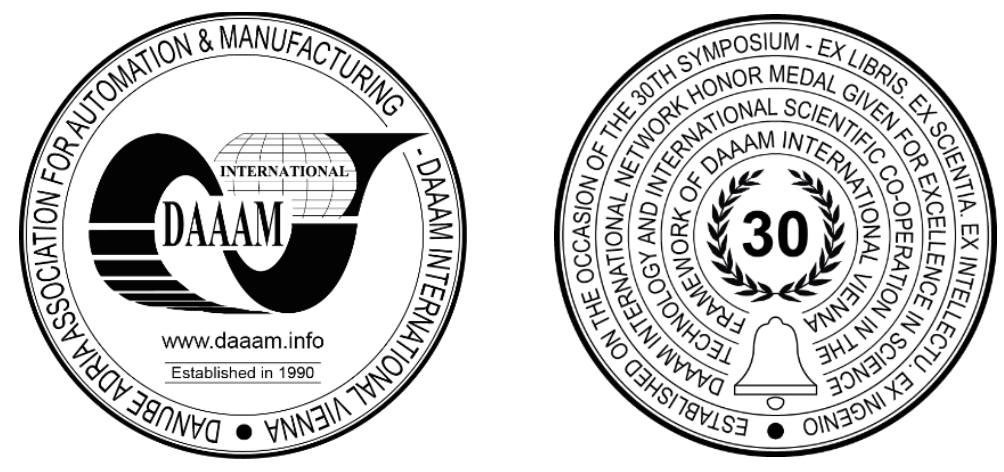

This Publication has to be referred as: Muster, L[ucas]; Woeber, W[ilfried] \& Aburaia, M[ohamed] (2021). Uniform Manufacturer-Independent Mobile Robot Programming, Proceedings of the 32nd DAAAM International Symposium, pp.0542-0545, B. Katalinic (Ed.), Published by DAAAM International, ISBN 978-3-902734-33-4, ISSN 1726-9679, Vienna, Austria

DOI: $10.2507 / 32$ nd.daaam.proceedings.078

\begin{abstract}
Reliability, flexibility and minimum processing time are the key production requirements for sustainably competitive manufacturing. A high degree of flexibility often requires adjustments to both the individual robot-operated systems and the mobile robots used on the process side, resulting in an automatic increase in setup times. A uniform manufacturerindependent programming method for robots would radically reduce programming time. This work describes the development and implementation of a programming concept for mobile robots which allows the use of uniform commands independent of the manufacturer. The resulting programming library breaks these commands down to a level where autonomous code can be generated for the individually used robot, eliminating the need for the user to deal with complex programming tools.
\end{abstract}

Keywords: Mobile robot; Programming library; Robot skills; Abstract programming

\section{Introduction}

To keep the adaptability in production lines as high as possible, a special focus is put on robotics, since it has become a decisive factor for years [4]. Due to the large number of different robot types, programming languages and applications, it is time consuming and difficult to create or adapt robot applications. Especially mobile robots are complex to program since they often have to rely on statistical algorithms [11]. If mobile robots are extended with an additional manipulator, a high degree of automation can be created [1]. 
However, this results in even greater complexity in the programming implementation of certain applications. Without a standard specification for robotic software the workload of programming is exceedingly high [2],[7]. Therefore, this work describes the development of an autonomous robot programming concept for a mobile robot. The contribution of this study is the development, implementation and evaluation of a generic skill-based library for mobile robot applications. This work is structured as follows. The next chapter describes the current state of the art. Then in chapter 3 the used methods are described. The results are presented in chapter 4. Finally, this paper is summarized in chapter 5.

\section{State of the Art}

In order to be able to design an abstract programming concept, a systematic model must be created, which lies above the actual programming of the robot. A possible implementation of this model can be done with a decision system, a state machine for the individual operations in the process, an operating system, reflecting the functions of the existing robot such as the control of a motor and the reading of a sensor and an information system, processing all forms of internal and external communication [3]. As an approach for the decision system and thus the generation of robot sequence programs can be designed with the help of Planning Domain Definition Language (PDDL) [13]. By determining so-called domain files for predicates, actions and problem files for objects, initial states and goal specifications, a certain procedure and code can be generated, providing the solution to a specific problem. In retrospect, a process can thus be changed very easily by integrating new states or constrains, for example, since the code or sequences generate themselves.

To implement all the functions of the robot, a certain sequence must also be generated in the software system. It is necessary to create a state machine for the individual commands in a way that the program can handle all kinds of functions, which can be divided into smaller commands, as shown by [10].

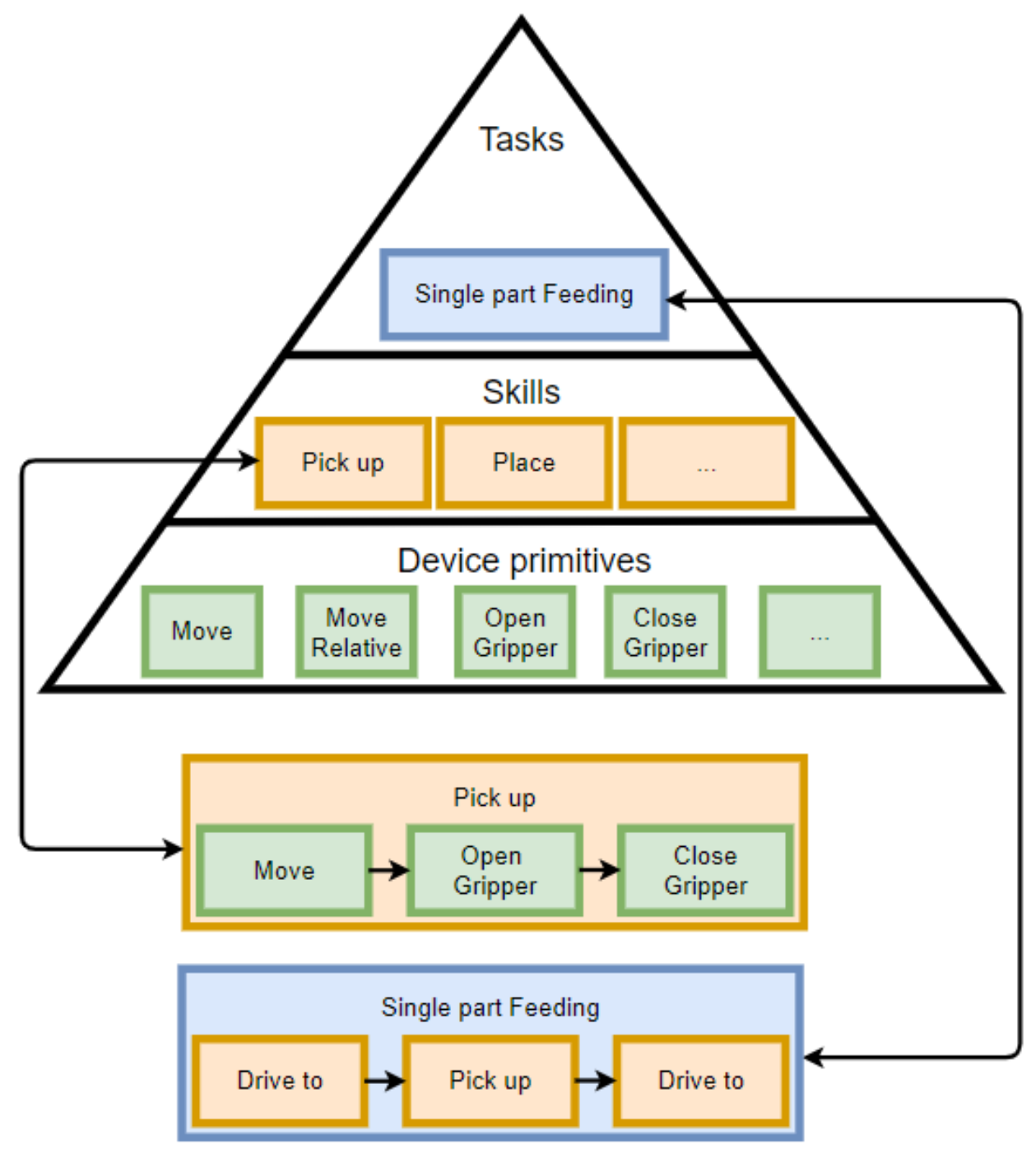

Fig. 1. Separation of individual functions into sub-functions [10]. The blue boxes, individual tasks defined by the user, are divided into smaller skills (yellow boxes), which in turn are divided into device primitives (green boxes). Thus, larger tasks can be divided into a sequence of smaller functions. 


\section{Materials and Methods}

Basic commands must be identified and described a prerequisite for all methods. Cartesian (robot-independent) motions, object manipulation or status reports are typical functions every robot must have included, and which are required as basic functions. The Canonical Robot Command Language (CRCL) is used for this purpose [8]. It describes all robot programming commands that are necessary to execute any applications, implemented. Furthermore, internal parameters like speed or tolerances are passed and adapted if necessary. In this work the library is integrated to the MiR100 [5] mobile robot which is used as a transport robot and is connected to an industrial computer. The manufacturer of the robot provides a REST communication, where the required information is sent or requested to the robot via commands.

Parameter that are not changeable via the REST communication must be processed over the Robot Operating System (ROS) framework [9]. In order to be able to implement all the functions of the robot, a certain sequence must be generated in the software system. These functions are divided into smaller steps, so that the direct function of the robot can be used [7]. The step-by-step structure of the individual functionalities is shown in figure 1 [7]. A top-down variant is shown, starting at the top with the process task, which is specified by the user and divided into individual skills. These skills are again formed into device primitives.

\section{Results and Discussion}

The proposed architecture was tested in a test factory of the UAS Technikum Wien [6] for transport and pick-and place tasks in a processing procedure for the mobile robot MiR100. Primarily, functions were tested, which were already defined by the REST API, such as driving to a defined position or docking to an integrated marker. If the mission is successfully created by the program as described above, it is passed to the mobile robot and will be executed. If many missions are created and saved one after the other, the mobile robot works through them in order until there are none left. Furthermore, the user can add additional actions to an already existing mission. The actions are first stored together with their priority and handed over to the robot. Based on this priority number, the sequence of actions in the mission is automatically defined and processed accordingly during testing.

A mission requiring the robot arm UR5 [12], needs an already existing program and can be called through this library as an own action. A sequence was tested where the mobile robot docks at a position and then a UR5 program is passed to the robot arm via the MiR100 link. Further tests were performed with different integrated actions to ensure a broad evaluation. However, no direct errors occurred, resulting in the mission and action being successfully sent to the robot and processed.

\section{Conclusion}

This work shows a robot programming concept and how it can be implemented. Therefore, to solve the problem of various types of robots with different applications and programming languages, such an abstract way of programming can be used in a complex manufacturing factory. Consequently, employees, who have no knowledge of robot programming can still implement new programs and missions in a quick and easy way. For technicians who are directly integrated in the process flow, the concept gives a possibility to change processes without having to read the specific robot applications and data sheets. As a result, time can be saved, especially in production since complex programs are not required.

In the further course of the development of this described library, additional functions will be integrated in order to implement any possible application with a mobile robot. Further tests and evaluations of the individual tasks and missions of the robot will be carried out. In addition, safety and security aspects have to be considered, as this is a critical factor especially in mobile robots.

This work has dealt exclusively with the abstract programming of the MiR100 mobile robot. To be able to program a complete production chain, including many types of industrial and mobile robots with different interfaces in, such a library has to be designed for each individual robot, which looks complicated in the first place, but offers clear advantages in the future.

\section{References}

[1] R. E. Andersen, E. B. Hansen, D. Cerny, S. Madsen, B. Pulendralingam, S. Bøgh, and D. Chrysostomou (2017). "Integration of a Skill based Collaborative Mobile Robot in a Smart Cyber-physical Environment," Procedia Manufacturing, vol. 11, no. October 2019, pp.114-123.

[2] Andreev, V[iktor]; Kim, V[alerii] \& Eprikov, S[tanislav] (2020). ModRob: the Hardware-Software Framework for Modular Mobile Robots Prototyping, Proceedings of the 31st DAAAM International Symposium, pp.0391-0402, B. Katalinic (Ed.), Published by DAAAM International, ISBN 978-3-902734- 29-7, ISSN 1726-9679, Vienna, Austria DOI: $10.2507 / 31$ st.daaam.proceedings.054 
[3] A.-L. Courbis, K. Luu, B. Grondin, and K. Roussel (2019). "A Model Driven Architecture Framework for Robot Design and Automatic Code Generation,” 15th China-Europe International Symposium on Software Engineering Education.

[4] G. Fragapane, D. Ivanov, M. Peron, F. Sgarbossa, and J. O. Strandhagen (2020). "Increasing flexibility and productivity in Industry 4.0 production networks with autonomous mobile robots and smart intralogistics," Annals of Operations Research.

[5] Mobile Industrial Robots A/S. (2019). MiR100 robot, [Online]. Available: https://www.mobile-industrialrobots.com/en/solutions/robots/mir100/.

[6] R. Otrebski, D. Pospisil, C. Engelhardt-Nowitzki, N. Kryvinska, and M. Aburaia (2019). "Flexibility enhancements in digital manufacturing by means of ontological data modeling," Procedia Computer Science, vol. 155, no. 2018, pp. 296-302.

[7] M. R. Pedersen, L. Nalpantidis, R. S. Andersen, C. Schou, S. Bøgh, V. Krüger, and O. Madsen (2020). "Robot skills for manufacturing: From concept to industrial deployment".

[8] F. Proctor, S. Balakirsky, Z. Kootbally, T. Kramer, C. Schlenoff, and W. Shackleford (2016). "The Canonical Robot Command Language (CRCL)," Industrial Robot, vol. 43, no. 5, pp. 495-502.

[9] M. Quigley, K. Conley, B. Gerkey, J. Faust, T. Foote, J. Leibs, R. Wheeler, and A. Ng (2009). "Ros: an open-source robot operating system," ICRA Workshop on Open Source Software, vol. 3.

[10] B. Ridge, T. Gaspar, and A. Ude (2017) "Rapid state machine assembly for modular robot control using metascripting, templating and code generation," IEEE-RAS International Conference on Humanoid Robots, pp. 661668.

[11] S. Thrun, W. Burgard, and D. Fox (2006). Probabilistic Robotics. MIT Press: Cambridge, MA, USA.

[12] Universal Robots. (2014). Technical Specifications UR5. Available at: http://www.universal-robots.com

[13] B. Wally, J. Vyskocil, P. Novak, C. Huemer, R. Sindelar, P. Kadera, A. Mazak, and M. Wimmer (2019). "Flexible Production Systems: Automated Generation of Operations Plans Based on ISA-95 and PDDL," IEEE Robotics and Automation Letters, vol. 4, no. 4, pp. 4062-4069. 